\title{
Lifetime risk of common neurological diseases in the elderly population
}

\author{
Silvan Licher, ${ }^{1}$ Sirwan K L Darweesh, ${ }^{1}$ Frank J Wolters, ${ }^{1,2}$ Lana Fani, ${ }^{1,2}$ \\ Alis Heshmatollah, ${ }^{1,2}$ Unal Mutlu, ${ }^{1,3}$ Peter J Koudstaal, ${ }^{2}$ Jan Heeringa, ${ }^{1}$ \\ Maarten J G Leening, 1,4,5 M Kamran Ikram, 1,2 M Arfan Ikram
}

\begin{abstract}
- Additional material is
published online only. To view please visit the journal online (http://dx.doi.org/10.1136/ jnnp-2018-318650).

${ }^{1}$ Department of Epidemiology, Erasmus MC - University Medical Center Rotterdam, Rotterdam, The Netherlands ${ }^{2}$ Department of Neurology, Erasmus MC - University Medical Center Rotterdam, Rotterdam, The Netherlands ${ }^{3}$ Department of Radiology and Nuclear Medicine, Erasmus MC - University Medical Center Rotterdam, Rotterdam, The Netherlands

${ }^{4}$ Department of Cardiology, Erasmus MC - University Medical Center Rotterdam, Rotterdam, The Netherlands ${ }^{5}$ Department of Epidemiology, Harvard T.H. Chan School of Public Health, Boston, Massachusetts, USA
\end{abstract}

\section{Correspondence to}

Dr M Arfan Ikram, Department of Epidemiology, Erasmus MC University Medical Center Rotterdam, Rotterdam 3015 GD The Netherlands; m.a.ikram@ erasmusmc.nl

SL, SKLD and FJW contributed equally.

MKI and MAI are joint last authors.

Received 18 April 2018 Revised 28 June 2018

Accepted 2 October 2018

Published Online First 2 October 2018

\section{Check for updates}

(C) Author(s) (or their employer(s)) 2019. No commercial re-use. See rights and permissions. Published by BMJ.

To cite: Licher S, Darweesh SKL, Wolters FJ, et al. J Neurol Neurosurg Psychiatry

2019:90:148-156.

\section{ABSTRACT}

Objective To quantify the burden of common neurological disease in older adults in terms of lifetime risks, including their co-occurrence and preventive potential, within a competing risk framework.

Methods Within the prospective population-based Rotterdam Study, we studied lifetime risk of dementia, stroke and parkinsonism between 1990 and 2016. Among 12102 individuals (57.7\% women) aged $\geq 45$ years free from these diseases at baseline, we studied co-occurrence, and quantified the combined, and disease-specific remaining lifetime risk of these diseases at various ages for men and women separately. We also projected effects on lifetime risk of hypothetical preventive strategies that delay disease onset by 1, 2 and 3 years, respectively.

Results During follow-up of up to 26 years (156088 person-years of follow-up), 1489 individuals were diagnosed with dementia, 1285 with stroke and 263 with parkinsonism. Of these individuals, 438 (14.6\%) were diagnosed with multiple diseases. Women were almost twice as likely as men to be diagnosed with both stroke and dementia during their lifetime. The lifetime risk for any of these diseases at age 45 was $48.2 \%$ (95\% Cl $47.1 \%$ to $51.5 \%$ ) in women and $36.2 \%$ (35.1\% to $39.3 \%)$ in men. This difference was driven by a higher risk of dementia as the first manifesting disease in women than in men $(25.9 \%$ vs $13.7 \% ; p<0.001)$, while this was similar for stroke (19.0\%vs $18.9 \%$ in men) and parkinsonism (3.3\% vs 3.6\% in men). Preventive strategies that delay disease onset with 1 to 3 years could theoretically reduce lifetime risk for developing any of these diseases by $20 \%-50 \%$.

Conclusion One in two women and one in three men will develop dementia, stroke or parkinsonism during their life. These findings strengthen the call for prioritising the focus on preventive interventions at population level which could substantially reduce the burden of common neurological diseases in the ageing population.

\section{INTRODUCTION}

Dementia, stroke and parkinsonism are among the leading causes of mortality and disability in older individuals and pose a huge burden on patients and their caregivers. ${ }^{1}$ These common neurological diseases share many risk factors and subsequently tend to show substantial co-occurrence, with stroke and parkinsonism patients at increased risk of dementia and patients with dementia at increased risk of stroke. ${ }^{23}$ Recent estimates indicate that the global costs-of-illness for these diseases sum up to more than $2 \%$ of the annual world gross domestic product. $^{4-6}$ This socioeconomic burden is expected to grow steeply with the ageing of populations and continuing increases in life-expectancy worldwide. ${ }^{1}$ As a result, this has led to widespread calls for prioritising these diseases on the global health agenda. ${ }^{7-9}$ Yet, these common neurological diseases remain understudied in terms of prevention at the population level ${ }^{1011}$ and underfunded compared with other common diseases such as cancer and heart disease, which likely reflects skewed societal perceptions of lifetime risk. ${ }^{12}{ }^{13}$ While informative risk estimates exist to characterise the burden of cancer and heart disease in the population, these numbers are lacking for these common neurological diseases. Such numbers are particularly suited to use in health campaigns to raise public awareness, with the lifetime risk of one in eight for breast cancer and the risk of one in four to die from heart disease as two key examples. ${ }^{14} 15$

Prior studies estimating the burden of these neurological diseases, relied on prevalence or incidence rates, without appropriately accounting for their potential co-occurrence and competing non-neurological mortality. ${ }^{14-6}$ To prevent potential overestimation, lifetime risk estimates can be used to reliably estimate this burden while taking into account the substantial co-occurrence of these diseases at old age, ${ }^{16}$ a high competing risk of mortality as well as potential differences in life-expectancy between men and women. These lifetime risk estimates are needed as these may benefit the awareness of the burden in the population and subsequently can be used to model what impact preventive interventions could have on this disease burden. ${ }^{8} 917-19$

In this study, we used long-term follow-up data from the population based Rotterdam Study to study occurrence and co-occurrence of dementia, stroke and parkinsonism in middle-aged and elderly men and women. We additionally calculated the corresponding combined, and disease-specific lifetime risk of these diseases within a competing risk framework, and studied their preventive potential.

\section{METHODS}

Study design, setting and population

This study was performed within the Rotterdam Study, a prospective population-based cohort designed to study the occurrence and determinants 
of age-related diseases in the general population. Details regarding the objectives and design have been reported previously. ${ }^{16}$ Briefly, in 1990, all inhabitants aged 55 and older from a well-defined suburb in the city of Rotterdam, the Netherlands. This initial cohort compromised 7983 individuals. In 2000, 3011 individuals who had become 55 years of age or moved into the study district since the start of the study if aged 55 years and older were added to the cohort. In 2006, a further extension of the cohort was initiated in which 3932 individuals were included, aged 45 years and older. In total, the Rotterdam Study comprises 14926 individuals aged 45 years or over. The overall response rate for all three recruitment waves was $72 \%$.

Because the aim was to determine the risk of developing disease, we excluded individuals who already had a history of these diseases at baseline $(n=1019$ : dementia $(n=420)$, stroke $(n=378)$, parkinsonism $(n=106)$ or with a history of a combination of these diseases $(n=115))$. We additionally excluded individuals who were insufficiently screened at baseline for at least one of these diseases $(n=1780)$. We further excluded individuals who did not provide informed consent to access medical records or hospital discharge letters $(\mathrm{n}=25)$, leaving 12102 individuals available for analyses.

\section{Ascertainment methods of dementia, stroke and parkinsonism}

Baseline and follow-up ascertainment methods for dementia, stroke and parkinsonism have previously been described in detail and are summarised in online supplementary appendix A. At baseline, disease ascertainment comprised extensive structured interviews, examinations and information from medical records, hospital discharge letters and pharmacy data to ensure that participants were free of any of these diseases. During follow-up, we screened for these diseases during repeated examinations and interviews every 4 years. We additionally ensured continuous monitoring for disease through computerised linkage of medical records from general practitioners and the regional institute for outpatient mental healthcare with the study database. In the Dutch healthcare system, the entire population is entitled to primary care that is covered by their obligatory health insurance. The general practitioner functions as a gatekeeper for referral to secondary and tertiary care providers, who report back to the referring general practitioner about test results and clinical diagnoses. With this linkage, the entire cohort is thus continuously monitored for detection of new cases or possible clinical signs of these diseases between centre visits.

Of all individuals who were screened and suspected of having any of these diseases, case reports were drawn up covering all potentially relevant information to establish the presence of disease. These case reports were evaluated by a consensus panel led by a consultant neurologist to adjudicate the final diagnosis in accordance with standardised diagnostic criteria which were held constant over the entire follow-up time DSM-III-R for all-cause dementia and NINCDS-ADRDA for Alzheimer's disease. Stroke was defined according to WHO criteria. Parkinson's disease', were defined according to strict study criteria (additional information in online supplementary appendix A). In addition, available clinical neuroimaging data were used if required to determine the subtype of diagnosis. The subtype of stroke was classified as unspecified if no imaging was available. Study follow-up ended at incident outcome diagnosis, death or 1 January 2016, whichever came first. Follow-up was virtually complete ( $96.2 \%$ of potential person-years).
Ascertainment methods of study population characteristics

Standardised assessment of anthropometrics, risk factors and use of medication at baseline through in-person examinations at the research centre, home interviews and laboratory assessments is described in online supplementary appendix B.

\section{Statistical analysis}

Preclusion of disease-specific outcomes of interest by death or precluding events is referred to as competing risks. ${ }^{20}$ Due to these competing events, absolute risks are overestimated in standard Kaplan-Meier analyses. Since women on average live longer than men, this overestimation will be differential. To overcome these issues, we analysed the data taking into account the occurrence of competing events to compute remaining lifetime risks in left truncated data with age as time scale, while stratifying by sex in all analyses. Lifetime risk estimates reflect the competing risk-adjusted cumulative incidences from that particular age to the age of last observation. In this study, the maximum age was 106 years for men and 107 years for women.

First, we studied occurrence and the co-occurrence of these diseases during follow-up. We quantified the number of events for each disease separately and visualised their co-occurrence among individuals using Venn diagrams.

Second, we calculated the combined and disease-specific lifetime risk. For these analyses, follow-up started at study entry (with the age of 45 years as minimum) and ended at the first date of diagnosis of any of the three diseases. This meant that we considered only the event of the three potential outcomes, whichever occurred first. For instance, individuals who first experienced a stroke during follow-up were no longer at risk for dementia or parkinsonism. Within this framework, we assessed the combined cumulative incidence, or risk, of these diseases from the age of 45 to the age of last observation. This equals the combined remaining lifetime risk of developing any of these diseases at the age of 45 . We subsequently repeated this analysis for each disease separately, resulting in disease-specific lifetime risks of first manifestation.

Third, we repeated these analyses by changing the age of entry (from 45 to 55, 65, 75 and 85 years and older), to study whether the disease-specific lifetime risks of first manifestation differed across various ages.

Fourth, we studied disease-specific lifetime risk by considering only the disease of interest as outcome, such that individuals remained at risk of these three diseases irrespective of the occurrence of a first event. This meant that individuals with a stroke or parkinsonism during follow-up in the dementia analysis, remained at risk for dementia.

Fifth, we repeated these analyses considering only the most common subtypes for each disease separately (including Alzheimer's disease and vascular dementia, ischaemic, haemorrhagic and unspecified stroke and Parkinson's disease).

Finally, we modelled the effects of a delay in disease onset on the combined lifetime risk of any disease and for each disease separately at index ages $45,55,65,75$ and 85 by postponing the date of diagnosis of all three diseases with 1, 2 and 3 years. In these analyses, we assumed a constant life-expectancy.

We used nominal significance levels to compare age and sex differences $(p<0.05)$. Data were handled and analysed with SPSS Statistics V.24.0.0.1 (IBM, Armonk, New York, USA) and R, CRAN V.3.4.3 (rms, etm and cmprsk packages).

This study is registered with the Netherlands National Trial Register and WHO International Clinical Trials Registry Platform under the shared catalogue number NTR6831. 


\begin{tabular}{|c|c|c|c|}
\hline & $\begin{array}{l}\text { All individuals } \\
(n=12 \text { 102) }\end{array}$ & $\begin{array}{l}\text { Men } \\
(n=5120)\end{array}$ & $\begin{array}{l}\text { Women } \\
(\mathrm{n}=6982)\end{array}$ \\
\hline Age in years, mean (SD) & $64.4(9.4)$ & $63.8(8.8)$ & $64.9(9.8)$ \\
\hline \multicolumn{4}{|l|}{ Educational level, n (\%) } \\
\hline Primary & $1943(16.1 \%)$ & $577(11.3 \%)$ & $1366(19.6 \%)$ \\
\hline Lower & $4820(39.8 \%)$ & $1494(29.2 \%)$ & $3326(47.6 \%)$ \\
\hline Further & $3306(27.3 \%)$ & $1844(36.0 \%)$ & $1462(20.9 \%)$ \\
\hline Higher & $1848(15.3 \%)$ & $1144(22.3 \%)$ & $704(10.1 \%)$ \\
\hline \multicolumn{4}{|l|}{ Smoking, $\mathrm{n}(\%)$} \\
\hline Never & $3894(32.2 \%)$ & $723(14.1 \%)$ & $3171(45.4 \%)$ \\
\hline Past & $5256(43.4 \%)$ & $2898(56.6 \%)$ & $2358(33.8 \%)$ \\
\hline Current & $2871(23.7 \%)$ & $1476(28.8 \%)$ & $1395(20.0 \%)$ \\
\hline $\begin{array}{l}\text { Systolic blood pressure in mm } \\
\mathrm{Hg} \text {, mean (SD) }\end{array}$ & $138(22)$ & $139(21)$ & $137(22)$ \\
\hline $\begin{array}{l}\text { Diastolic blood pressure in } \mathrm{mm} \\
\mathrm{Hg} \text {, mean (SD) }\end{array}$ & $77(12)$ & $78(12)$ & $76(12)$ \\
\hline Hypertension, $\mathrm{n}(\%)$ & $6481(53.6)$ & $2744(53.6)$ & $3737(53.5)$ \\
\hline Depression, n (\%) & $801(6.6 \%)$ & $195(3.8 \%)$ & $606(8.7 \%)$ \\
\hline Atrial fibrillation, $\mathrm{n}(\%)$ & $599(4.9 \%)$ & $309(6.0 \%)$ & $290(4.2 \%)$ \\
\hline Type 2 diabetes, n (\%) & $1124(9.3 \%)$ & $574(11.2 \%)$ & $550(7.9 \%)$ \\
\hline Total cholesterol, mmol/L & $6.18(1.24)$ & $5.91(1.18)$ & $6.39(1.24)$ \\
\hline $\begin{array}{l}\text { High-density lipoprotein } \\
\text { cholesterol, mmol/L }\end{array}$ & $1.38(0.39)$ & $1.22(0.33)$ & $1.49(0.40)$ \\
\hline Hypercholesterolaemia, n (\%) & $5489(45.4)$ & $1842(36.0)$ & $3647(52.2)$ \\
\hline \multicolumn{4}{|l|}{ APOEgenotype, $\mathrm{n}(\%)$} \\
\hline$\varepsilon 2 / \varepsilon 2$ or $\varepsilon 2 / \varepsilon 3$ & $1510(12.5 \%)$ & $605(11.8 \%)$ & $905(13.0 \%)$ \\
\hline$\varepsilon 3 / \varepsilon 3$ & $6675(55.2 \%)$ & $2874(56.1 \%)$ & $3801(54.4 \%)$ \\
\hline$\varepsilon 2 / \varepsilon 4, \varepsilon 3 / \varepsilon 4$ or $\varepsilon 4 / \varepsilon 4$ & $3239(26.8 \%)$ & $1400(27.3 \%)$ & $1839(26.4 \%)$ \\
\hline
\end{tabular}

\section{RESULTS}

Population characteristics are presented in table 1. Median age at baseline was 62.2 years (range 45-107 years), and women represented $57.7 \%$ of the population. During 156088 person-years of follow-up, 3037 events occurred: 1489 individuals were diagnosed with dementia (79.7\% Alzheimer's disease), 1285 with stroke $(64.7 \%$ ischaemic, 9.8\% haemorrhagic stroke and 25.4\% unspecified stroke) and 263 with parkinsonism (50.6\% Parkinson's disease). A complete overview of the occurrence of all subtypes of disease is presented in online supplementary table 1 in appendix C. In total, 5291 individuals died during follow-up, of whom 3260 died free of these diseases. Individuals who were diagnosed with one of these diseases during follow-up, particularly tended to have a higher prevalence of hypertension $(61.5 \%$ vs $51.4 \%)$ atrial fibrillation $(7.7 \%$ vs $4.2 \%)$, hypercholesterolemia (56.0\% vs $42.5 \%)$ and more often had type 2 diabetes $(11.2 \%$ vs $8.8 \%)$ at baseline, compared with those who were not diagnosed with any of these diseases during follow-up (online supplementary table 2, appendix C).

\section{Disease co-occurrence}

Among those who developed one of these three neurological diseases, most individuals were diagnosed with only one of these diseases (figure 1). Yet, there is a substantial risk at the age of 45 to face multiple diseases during the remaining lifespan. Among those diagnosed with one of these neurological diseases, 438 $(14.6 \%)$ individuals were diagnosed with more than one disease, with women more likely to be diagnosed with more than one of these diseases during lifetime compared with men (figure 1:4.0\%

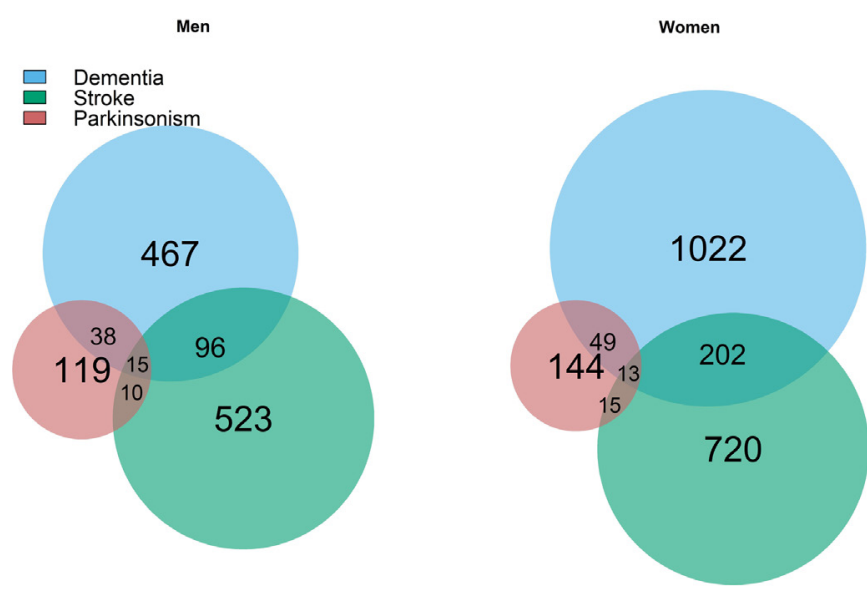

Figure 1 Venn diagrams showing patterns of disease co-occurrence in men and women quantified in the number of events during follow-up. Of all women, $4 \%$ were diagnosed with more than one of these diseases, whereas for men this approximated $3 \%$.

compared with $3.1 \%$, respectively, $\mathrm{p}<0.001)$. This difference was predominantly driven by a greater probability of overlap between dementia and stroke (occurring in $2.9 \%$ of all women compared with $1.9 \%$ in men, $\mathrm{p}<0.001$ ).

\section{Lifetime risk of any disease}

Among all individuals free of dementia, stroke and parkinsonism at baseline ( $\mathrm{N}=12$ 102), a total of 2571 individuals were diagnosed with one of these three diseases as first manifestation: 1245 were diagnosed with dementia, 1118 individuals with stroke and 208 with parkinsonism. In figure 2, the combined cumulative risk of developing any of these diseases from the age of 45 until various ages is presented for women and men separately. This risk increased steeply with age, ranging from $2.6 \%$ for women and $3.2 \%$ for men aged 45 years until age 65 , to up to $45.8 \%$ and $35.3 \%$ until the age 95 , respectively. The overall remaining lifetime risk of developing any of these diseases for a 45 -year-old woman was $48.2 \%$ (95\% CI $47.1 \%$ to $51.5 \%$ ), while for a 45 -year-old man this risk was $36.3 \%$ (35.1\% to $39.3 \%$, p for sex difference $<0.001$ ).

\section{Lifetime risk of any disease across age}

Overall lifetime risk of these common neurological diseases was stable for both men and women between ages 45 and 85 (figure 3). At age 45, first manifestation of stroke posed the highest lifetime risk for men (18.9\%). Dementia posed the largest risk for women (25.9\%), which was significantly higher compared with that for men $(13.7 \%$; $p$ for sex difference $<0.001)$. This sex difference remained largely stable across all index ages $(p<0.001$ for all index ages). Figure 3 also shows that with advancing age, the relative contribution of dementia to the remaining lifetime risk of any disease increased in both men and women, representing $66.6 \%$ of all first diagnoses in elderly women (ie, $>85$ years) and $55.6 \%$ in elderly men. For stroke, men and women had a similar lifetime risk at the age of 45 (18.9\% in men compared with $19.0 \%$ in women, $\mathrm{p}=0.46)$. However, men were at substantially higher risk of developing stroke at younger ages, such that they have a $8.4 \%$ risk of developing stroke before the age of 75 years compared with $5.8 \%$ for women $(p=0.005)$. Lifetime risk of parkinsonism peaked earlier compared with dementia and stroke, was low after 85 years and 


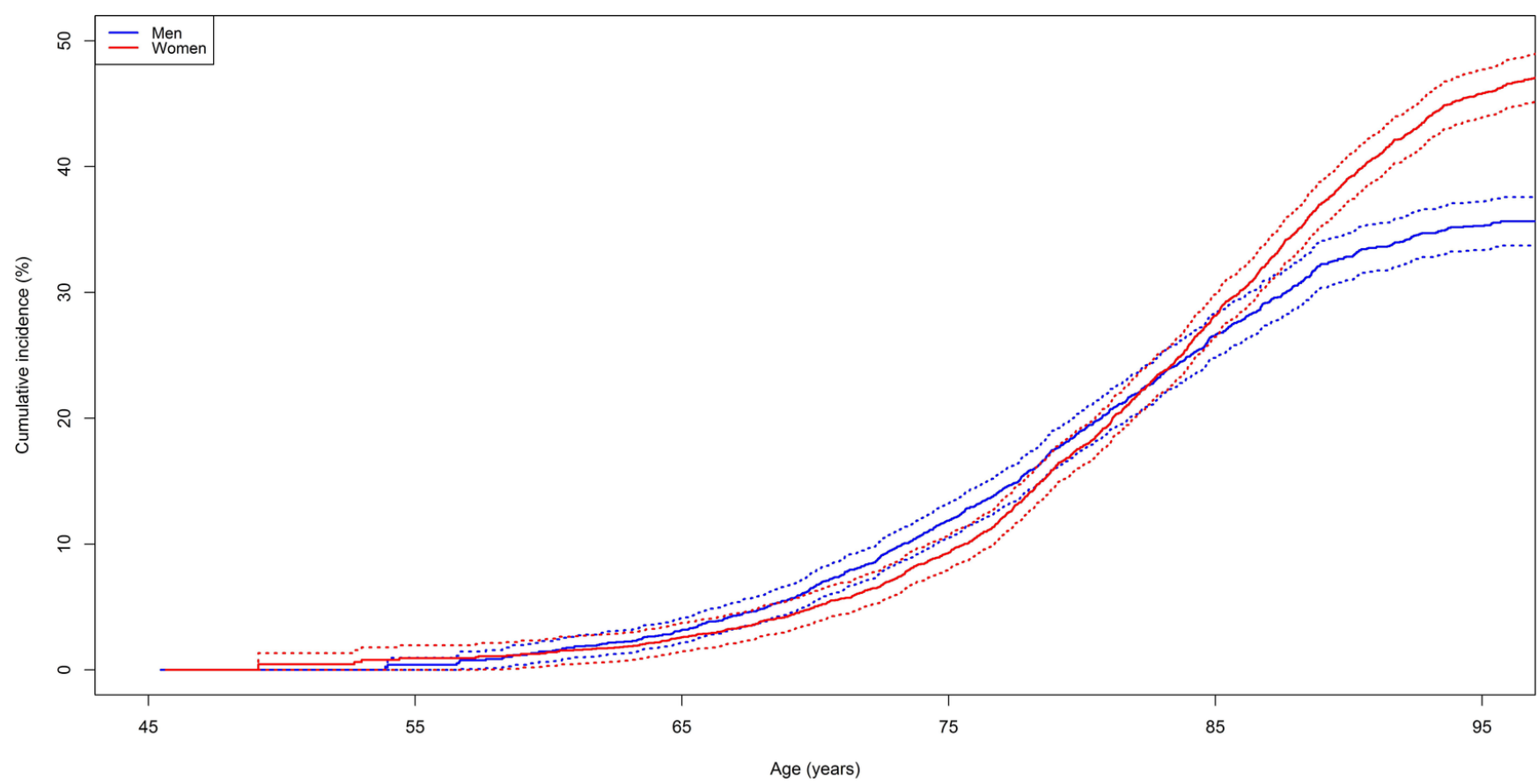

Figure 2 Risk of common neurological diseases for 45-year-old men and women. In this analysis, follow-up ended at time of first occurrence of dementia, stroke or parkinsonism. For instance, for individuals who first suffered a stroke and subsequently developed dementia, only the stroke event is considered here.

not significantly different between men and women at any age $(\mathrm{p}>0.16)$.

\section{Lifetime risk of each disease separately}

When individuals remained at risk of all diseases irrespective of the occurrence of precluding events, women aged 45 years had a significantly higher lifetime risk of developing dementia and stroke than men $31.4 \%$ compared with $18.6 \%$ in men; $\mathrm{p}<0.001$, and $21.6 \%$ compared with $19.3 \%$ in men; $\mathrm{p}=0.03$, respectively), whereas lifetime risk of parkinsonism (4.3\% in women and $4.9 \%$ in men, $\mathrm{p}=0.28$ ) was not significantly different (figure 4). We observed similar patterns in sex-specific occurrence for Alzheimer's disease and vascular dementia, ischaemic, haemorrhagic and unspecified stroke and Parkinson's disease (Appendix C, online supplementary figure 1 and table 3 ).

\section{Projecting a delay in disease onset and occurrence}

When projecting a delay in disease onset of 1,2 or 3years for all three diseases, the remaining lifetime risk of these common neurological diseases could be reduced by $20 \%$ in individuals aged 45 years and older, and by more than $50 \%$ in the oldest of old (figure 5). Even a delay in onset for a few years of only one disease could already result in substantial reductions for the combined lifetime risk of developing any of these diseases. For instance, delaying dementia onset with 3years has the potential to reduce lifetime risk of any disease by $15 \%$ for men and women aged 45 , up to $30 \%$ for those aged 85 years and older. For an 85 -year-old woman, this lowers the risk of developing dementia during her remaining lifetime from $30.4 \%$ to $21.3 \%$.

\section{DISCUSSION}

In this population-based study with long-term follow-up, we found that one in two women were diagnosed with dementia, stroke or parkinsonism during their lifetime, whereas for men this risk approximated one in three. Moreover, the risk of combined disease was higher in women than in men, with women almost twice as likely to be diagnosed with both stroke and dementia during their lifetime. These findings illustrate that lifetime risks of these diseases are very high. We further show that preventive strategies that delay disease onset of all three diseases by $1-3$ years have the potential to reduce these risks by $20 \%-50 \%$. These findings strengthen the call for focus on prevention to reduce the current and projected burden of common neurological diseases in the ageing population.

\section{Limitations and strengths}

Several methodological considerations should be taken into account when interpreting these lifetime risks. First, this population-based study predominantly included individuals of European ancestry (97\%), with a relatively long life-expectancy. ${ }^{21}$ Generalising these lifetime risk estimates to other ethnicities or to populations with different life-expectancies should therefore be done with caution. Second, although the overall response rate in the Rotterdam Study was high (72\%), non-responders and individuals with insufficient screening at study entry may have had higher than average risk factor burden and associated risk of these diseases, which may have led to some underestimation or overestimation of lifetime risks. ${ }^{22}$ The direction of this effect is balancing on the impact of such risk factor burden on disease risk on the one hand and the impact on the underlying life-expectancy of these individuals on the other hand. Third, we acknowledge that parkinsonism may not be recognised as a disease itself, instead it represents a spectrum of underlying pathologies, ranging, for example, from Parkinson's disease (representing $50.6 \%$ of all parkinsonism cases in this study) to more reversible causes of disease such as drug-induced parkinsonism (representing only 9.7\%). Nevertheless, considering such pathologies as disease is commonly done in public health reports, such as the WHO topic on the global burden of disease. Fourth, we only considered the first neurological disease that became 


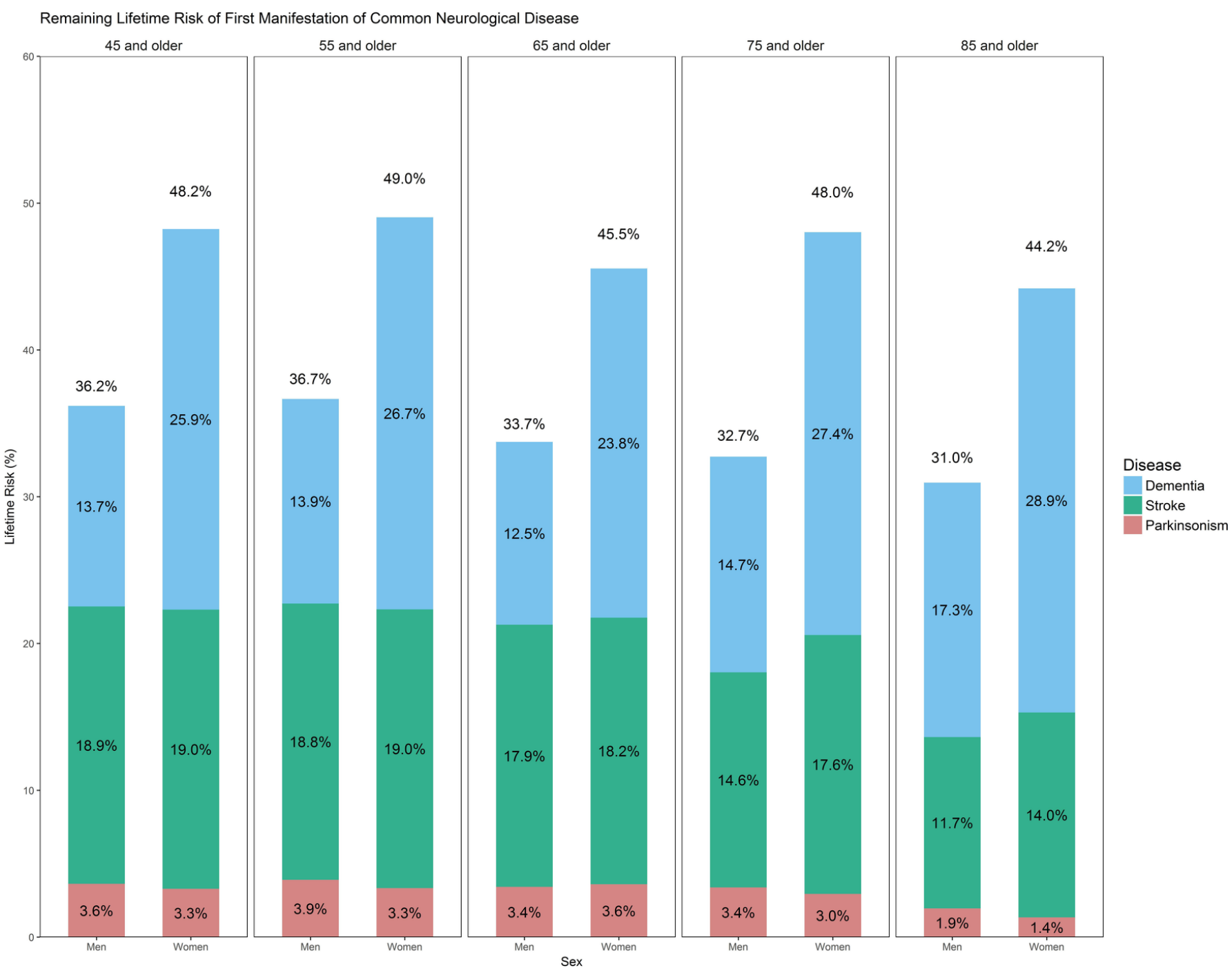

Figure 3 Remaining lifetime risk of first manifestation of common neurological diseases at different ages, stratified by sex.

clinically manifested in these analyses-this may have underestimated the hidden additional burden imposed by neurodegenerative diseases, which may extend beyond individuals with clinical dementia or parkinsonism, as long prodromal phases of these neurodegenerative diseases may have preceded a clinically overt stroke. Finally, we were unable to take into account severity of clinical disease, and in particular for interpretation of stroke estimates, it should be noted that about half of ischaemic strokes at the population level classify as minor according to the NIH Stroke Scale and may have a limited impact on daily life. ${ }^{23}$ Strengths of this study include the large sample size and simultaneous assessment of these diseases using virtually complete longterm follow-up data in the unselected general population.

\section{Previous studies}

So far, burden of these diseases in terms of lifetime risk were largely quantified separately ${ }^{24-29}$ or were determined based on prevalence or incidence rates, while not appropriately accounting for their potential co-occurrence and competing non-neurological mortality. ${ }^{1}$ This pattern of co-occurrence and competing mortality hampers reliable calculation of the risk of developing any of these diseases when applying a lifetime perspective, such that the occurrence of one disease (eg, stroke) precludes consideration of any subsequent event (eg, poststroke dementia).
Similarly, several risk factors (eg, hypertension) increase the susceptibility for these common neurological diseases and are associated with an increased risk of dying from other causes (eg, heart disease).

Previously, the Framingham Heart Study reported lifetime risks of both dementia ( 1 in 5 women, 1 in 10 men $)^{2629}$ and stroke ( 1 in 5 women, 1 in 6 men $)^{24}$ as well as a combined estimate of those two assessed in a single study (1 in 3). ${ }^{25}$ Compared with findings from that study, we observed higher a lifetime risk of dementia ( 1 in 3 women, 1 in 5 men). This discrepancy may be due to the fact that individuals in the Rotterdam Study, have a longer life-expectancy (the Netherlands, women 83.5 and men 81.7 years) compared with individuals in the Framingham Heart Study (USA, women 81.2 and men 76.4 years). Apart from a longer life-expectancy in general, these findings may be explained by smaller differences in life-expectancy between men and women in the Netherlands (1.8 years), compared with the USA (4.8 years). With longer life-expectancy, individuals in this study simply had more time to develop these diseases in a timeframe with high age-specific incidence rates. ${ }^{21}$ Additionally, women in this study were substantially lower educated compared with men, which may have led to a lower dementia resilience in women. In line with estimates from the Framingham Heart Study, we found a slightly higher lifetime risk of stroke for women 
Dementia

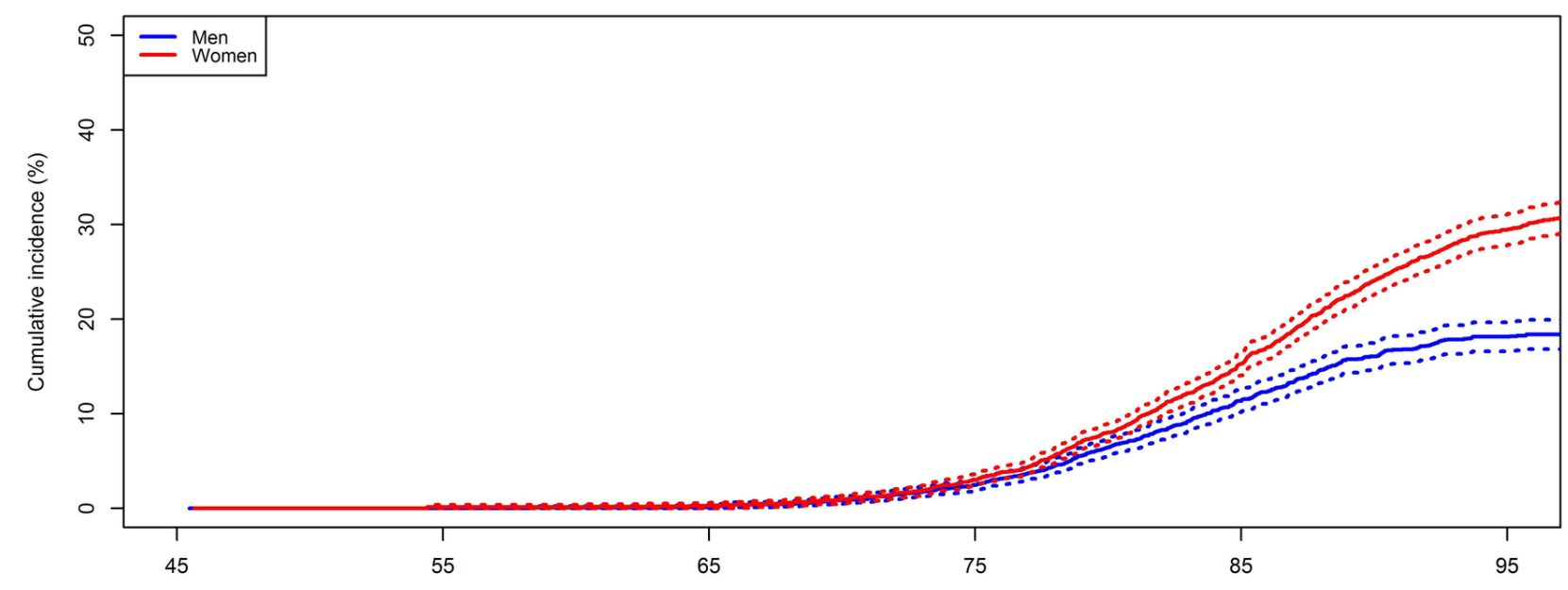

Stroke

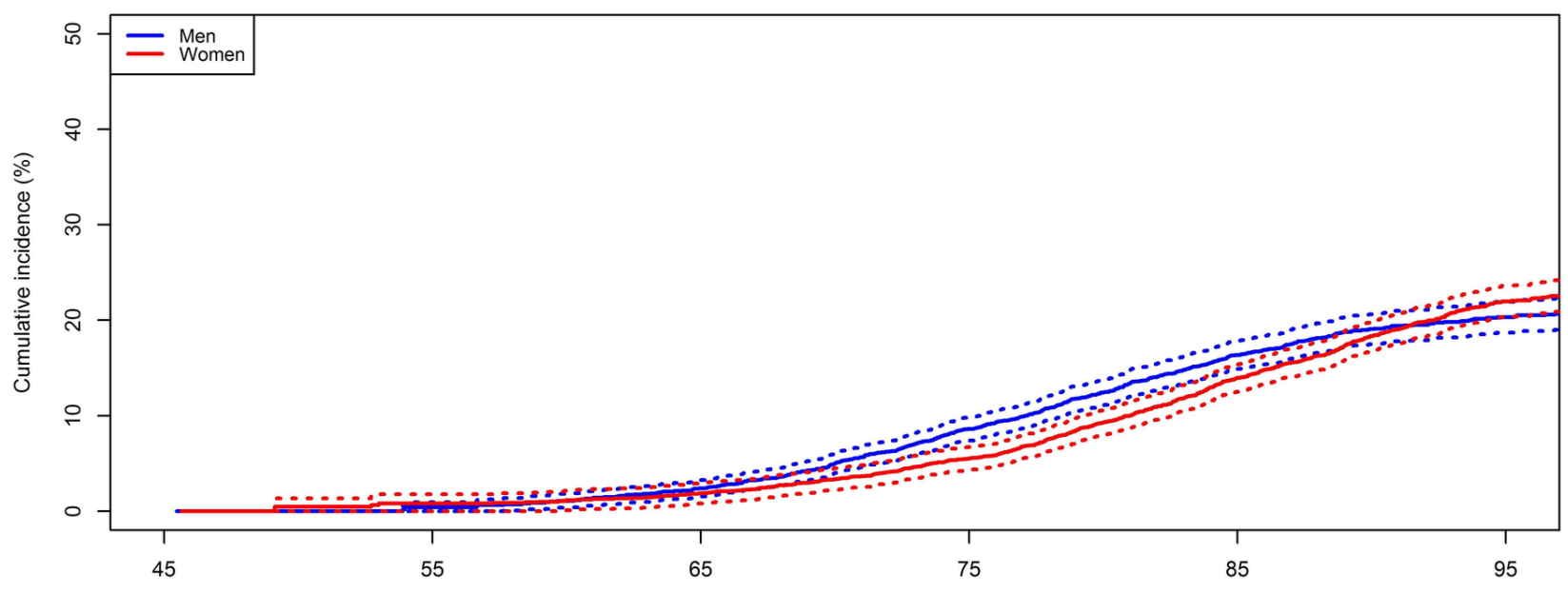

Parkinsonism

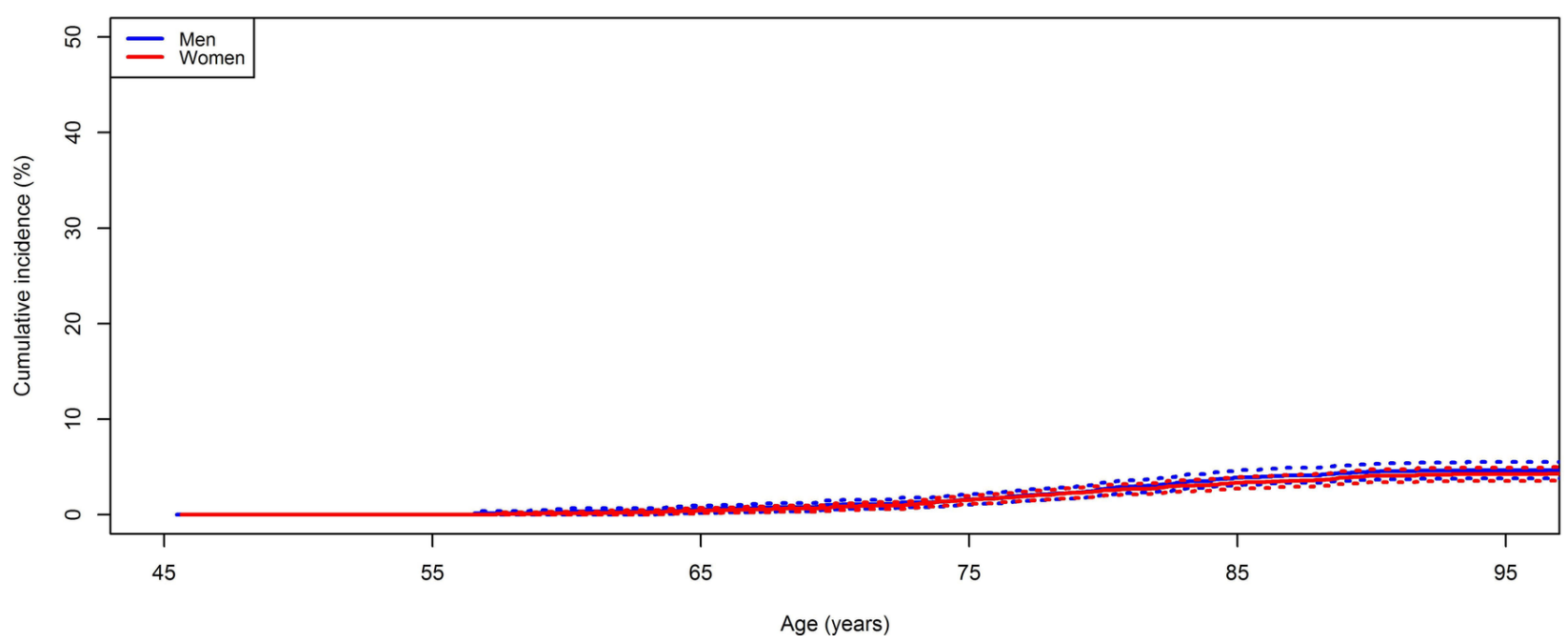

Figure 4 Risk of dementia, stroke and parkinsonism for 45-years-old men and women. In these analyses, individuals remained at risk of all diseases irrespective of the occurrence of precluding events. For instance, individuals with a stroke or parkinsonism during follow-up in the dementia analysis, remained at risk for dementia. Dotted lines represent 95\% Cls. 

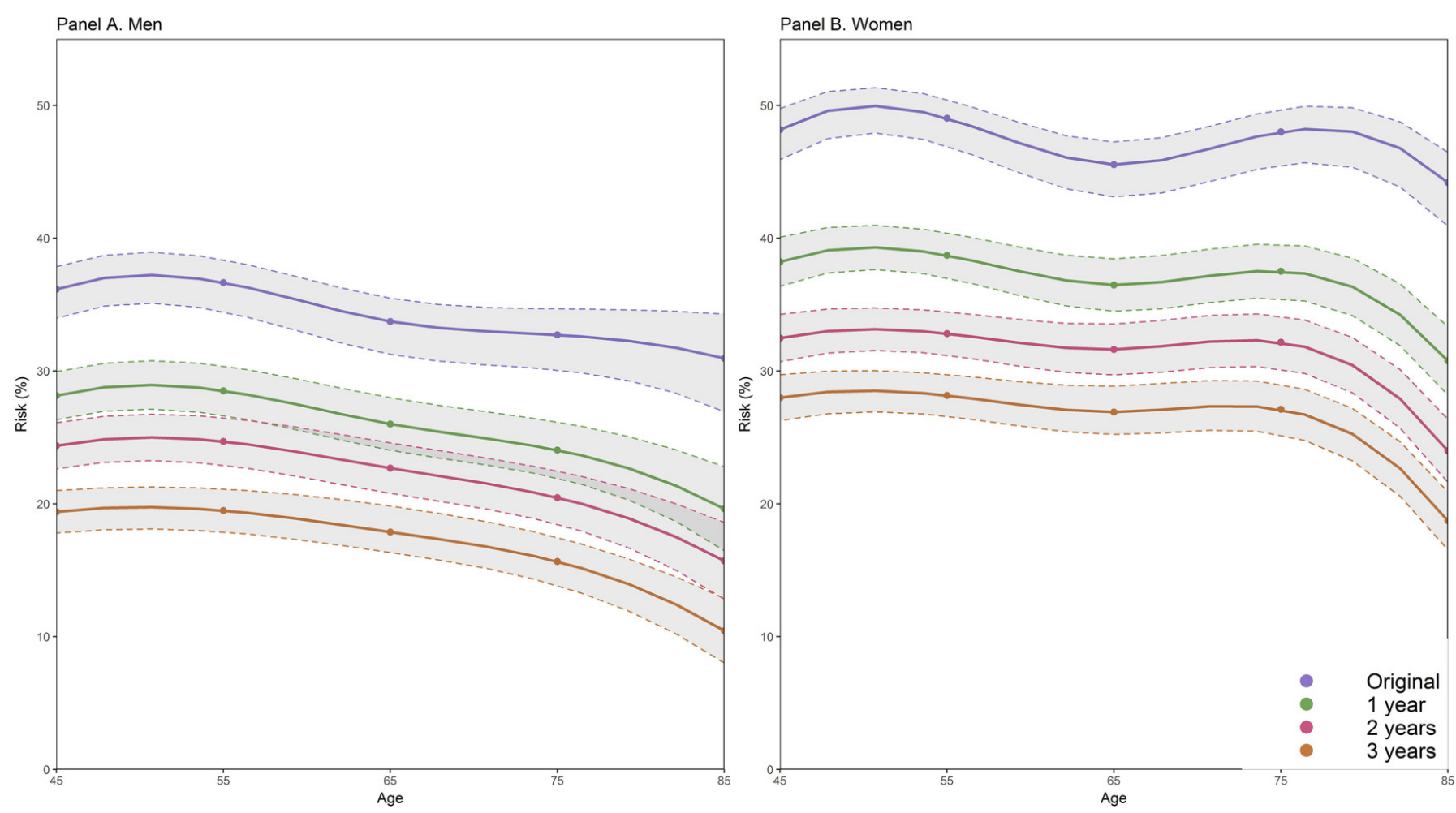

Figure 5 Modelling the effect of a 1, 2 and 3year delayed onset of disease on the combined remaining lifetime risk of developing dementia, stroke or parkinsonism in men (Panel A) and women (Panel B) at ages 45, 55, 65, 75 and 85 and older. In these analyses, date of diagnosis of all three diseases was delayed with 1, 2 or 3 years while assuming a constant life-expectancy. Areas in shaded grey represent $95 \% \mathrm{Cls}$.

compared with men. Although men have in general a greater propensity of developing a stroke before the age 90 , stroke rates for women are higher at the extremes of the age distribution with an increased risk of developing ischaemic stroke for those aged $<65$ years old and a higher risk of unspecified strokes for women among the oldest-old. Although men in this study had more adverse levels of stroke risk factors at baseline, recent evidence shows that stroke rates among women are catching up with those from men due to recent increases in the prevalence of several stroke risk factors, particularly among young women, including hypertension, smoking and drug abuse. ${ }^{30}$

For parkinsonism and Parkinson's disease, we found similar lifetime risks for men and women, corroborating evidence from a previous study. ${ }^{27}$ In contrast, we did find lower risks of Parkinson's disease for men (2.9\%), compared with the lifetime risk of $6.7 \%$ reported in another study solely conducted in male physicians. ${ }^{28}$ Physicians may be more aware of their changing health (eg, symptom recognition), which may help explain this higher risk. In addition, this discrepancy may be due to distinct differences in baseline characteristics, such as a lower history of eversmoking (50\% compared with $88 \%$ in this study), a factor that is inversely associated with Parkinson's disease. ${ }^{31}$

\section{Implications and future directions}

Lifetime risk estimates can be used to effectively inform policy makers and communicate risks to the general population, given their easier interpretation compared with measures such as incidence rates, prevalence or relative risk. ${ }^{32}{ }^{33}$ These approaches to raise awareness and inform the public through lifetime risk estimates have been successfully implemented for other diseases, such as breast cancer or heart disease. ${ }^{1415}$ Nowadays, preventive measures for primary prevention of cardiovascular disease are tailored to individual lifetime risk estimates. ${ }^{34}$ This could inform future prevention programme for common neurological diseases. For this public policy planning perspective, future studies with an even broader age span could expand on the current study by specifically targeting other common neurological diseases such as multiple sclerosis. This would provide insight into the lifetime risk of multiple sclerosis. Additionally, other common diseases in the elderly population could be included, such as polyneuropathy, to provide an even more complete picture of the burden of neurological disease in the community.

This study provided a descriptive and quantitative overview of the burden of three common neurological diseases in the elderly population. To extend this overview, lifetime risk estimates for these diseases across different ethnicities are warranted. Additionally, it would be of particular interest for future studies to further study the effects of (epi) genetic or lifestyle factors that could impact the lifetime risk of these diseases.

There are currently no disease modifying drugs available for dementia and most causes of parkinsonism, and prevention of stroke is hampered by suboptimal adherence to effective preventive strategies or unmet guideline thresholds. ${ }^{35}$ Yet, a delay in onset of these common neurological diseases by merely a few years could reduce the population burden of these diseases substantially. In fact, preventive interventions may also contribute to a drop in competing mortality by affecting lifetime risk of diseases with similar risk factors. For instance, preventive interventions resulting in a delay in onset of strokes are likely also able to delay morbidity and mortality due to coronary or peripheral artery disease. Our projections of the preventive potential, which assumed constant life-expectancy, may therefore be overestimating the compression of morbidity and must therefore be interpreted as the upper limit of this preventive potential. In particular with diseases that are most common in the elderly, increased life-expectancy due to for example risk factor control 
may counterbalance lowering in incidence rates. ${ }^{36}$ Nevertheless, they illustrate that risks could drop strikingly with relatively minor delays in the occurrence and onset of disease, underlining the importance of preventive strategies as the way forward to combat these diseases on a global scale. ${ }^{57-41}$ Recent observations on declining dementia incidence trends from several, large population-based studies in high-income countries may in fact reflect the (initial) signs of these preventive strategies through better vascular risk factor management, improved educational attainment or other public health developments that improved the resilience for dementia. ${ }^{42-44}$ These results also have implications for the type of prevention strategies to develop, including population-wide interventions targeted at risk factors with considerable sex-specific differences in attributable risk, such as loneliness and depression in women or diet in men. ${ }^{1938}$

\section{CONCLUSIONS}

One in two women develops dementia, stroke or parkinsonism in their lifetime, whereas this risk approximates one in three for men. Women are almost twice as likely as men to be diagnosed with both stroke and dementia during their lifetime. Risks are theoretically highly amendable by preventive interventions at the population level. These findings strengthen the call for focus on preventive interventions to reduce the burden of common neurological disease in the ageing population.

Acknowledgements We thank all staff at the Rotterdam Study research centre, facilitating assessment of participants throughout the years. We acknowledge the support of Frank J.A. van Rooij as data manager.

Contributors All authors have made a substantial intellectual contribution to design of the study (MKI, MAI), acquisition of data (PJK, MKI, JH, SKLD, FJW, UM, SL, $L F, A H$ ), analysis and interpretation of data (SKLD, FJW, SL, MKI, MAI), drafting the manuscript (SL, SKLD, FJW) or revising it critically for important intellectual content (PJK, LF, AH, UM, JH, MJGL, MKI, MAI). All authors approved the final version of the manuscript for publication. MAl had full access to the data in the study and takes responsibility for data integrity and accuracy of data analysis.

Funding The Rotterdam Study is sponsored by the Erasmus MC and Erasmus University Rotterdam, The Netherlands Organisation for Scientific Research (NWO), The Netherlands Organisation for Health Research and Development (ZonMw), the Research Institute for Diseases in the Elderly (RIDE), The Netherlands Genomics Initiative, the Ministry of Education, Culture and Science, the Ministry of Health, Welfare and Sports, the European Commission (DG XII) and the Municipality of Rotterdam. Further support was obtained from the Netherlands Consortium for Healthy Ageing and the Dutch Heart Foundation (2012T008). None of the funding organisations or sponsors were involved in study design, in collection, analysis and interpretation of data, in writing of the report or in the decision to submit the article for publication.

Competing interests None declared.

Patient consent Not required.

Provenance and peer review Not commissioned; externally peer reviewed.

Data sharing statement Because of ethical restrictions, data are available on request. Interested researchers may contact our data management team (secretariat. epi@erasmusmc.nl) or the corresponding author of this study.

\section{REFERENCES}

1 GBD 2015 Neurological Disorders Collaborator Group. Global, regional, and national burden of neurological disorders during 1990-2015: a systematic analysis for the Global Burden of Disease Study 2015. Lancet Neurol 2017;16:877-97.

2 Pendlebury ST, Rothwell PM, Prevalence RPM. Prevalence, incidence, and factors associated with pre-stroke and post-stroke dementia: a systematic review and metaanalysis. Lancet Neurol 2009:8:1006-18.

3 de Lau LM, Schipper CM, Hofman A, et al. Prognosis of Parkinson disease: risk of dementia and mortality: the Rotterdam Study. Arch Neurol 2005;62:1265-9.

4 Wimo A, Guerchet M, Ali GC, et al. The worldwide costs of dementia 2015 and comparisons with 2010. Alzheimers Dement 2017;13:1-7.

5 Feigin VL, Norrving B, Mensah GA. Global Burden of Stroke. Circ Res 2017:120:439-48.
6 Kowal SL, Dall TM, Chakrabarti R, et al. The current and projected economic burden of Parkinson's disease in the United States. Mov Disord 2013:28:311-8.

7 Norton S, Matthews FE, Barnes DE, et al. Potential for primary prevention of Alzheimer's disease: an analysis of population-based data. Lancet Neurol 2014;13:788-94

8 Hankey GJ, prevention Sstroke. Secondary stroke prevention. Lancet Neurol 2014;13:178-94

9 Brainin M, Feigin V, Martins S, et al. Cut stroke in half: Polypill for primary prevention in stroke. Int J Stroke 2018:174749301876119.

10 Brayne C, Davis D. Making Alzheimer's and dementia research fit for populations. Lancet 2012;380:1441-3.

11 Rothwell PM. Funding for practice-oriented clinical research. Lancet 2006:368:262-6.

12 Luengo-Fernandez R, Leal J, Gray A. UK research spend in 2008 and 2012: comparing stroke, cancer, coronary heart disease and dementia. BMJ Open 2015;5:e006648

13 NIH. 2018.NIH Research Portfolio Online Reporting Tools (RePORT) https://report.nih. gov/categorical_spending.aspx.

14 Breast Cancer Campaign (BCA). 2017. The Breast Cancer Campaign visit us this Fall $2018 \mathrm{http}: / /$ www.bcacampaign.com/.

15 Breakaway From Heart Disease. 2018.Break Away from Heart Disease Campaign https://www.breakawayfromheartdisease.com/.

16 Ikram MA, Brusselle GGO, Murad SD, et al. The Rotterdam Study: 2018 update on objectives, design and main results. Eur J Epidemiol 2017;32:807-50.

17 de Bruijn RF, Bos MJ, Portegies ML, et al. The potential for prevention of dementia across two decades: the prospective, population-based Rotterdam Study. BMC Med 2015;13:132.

18 O'Donnell MJ, Chin SL, Rangarajan S, et al. Global and regional effects of potentially modifiable risk factors associated with acute stroke in 32 countries (INTERSTROKE): a case-control study. Lancet 2016;388:761-75.

19 Bos MJ, Koudstaal PJ, Hofman A, et al. Modifiable etiological factors and the burden of stroke from the Rotterdam study: a population-based cohort study. PLoS Med 2014:11:e1001634.

20 Satagopan JM, Ben-Porat L, Berwick $M$, et al. A note on competing risks in survival data analysis. Br J Cancer 2004;91:1229-35.

21 WorldBank. 2017.Data Worldbank https://data.worldbank.org/indicator/SP.DYN.LE00. IN.

22 Leening MJ, Heeringa J, Deckers JW, et al. Healthy volunteer effect and cardiovascular risk. Epidemiology 2014;25:470-1.

23 Reeves M, Khoury J, Alwell K, et al. Distribution of National Institutes of Health stroke scale in the Cincinnati/Northern Kentucky Stroke Study. Stroke 2013:44:3211-3.

24 Seshadri S, Beiser A, Kelly-Hayes M, et al. The lifetime risk of stroke: estimates from the Framingham Study. Stroke 2006;37:345-50.

25 Seshadri S, Wolf PA. Lifetime risk of stroke and dementia: current concepts, and estimates from the Framingham Study. Lancet Neurol 2007;6:1106-14.

26 Seshadri S, Wolf PA, Beiser A, et al. Lifetime risk of dementia and Alzheimer's disease. The impact of mortality on risk estimates in the Framingham Study. Neurology 1997;49:1498-504.

27 Elbaz A, Bower JH, Maraganore DM, et al. Risk tables for parkinsonism and Parkinson's disease. J Clin Epidemiol 2002;55:25-31.

28 Driver JA, Logroscino G, Gaziano JM, et al. Incidence and remaining lifetime risk of Parkinson disease in advanced age. Neurology 2009;72:432-8.

29 Chêne G, Beiser $A, A u R$, et al. Gender and incidence of dementia in the Framingham Heart Study from mid-adult life. Alzheimers Dement 2015;11:310-20.

30 George MG, Tong X, Bowman BA. Prevalence of cardiovascular risk factors and strokes in younger adults. JAMA Neurol 2017;74:695-703.

31 Hernán MA, Zhang SM, Rueda-deCastro AM, et al. Cigarette smoking and the incidence of Parkinson's disease in two prospective studies. Ann Neurol 2001;50:780-6.

32 Fortin JM, Hirota LK, Bond BE, et al. Identifying patient preferences for communicating risk estimates: a descriptive pilot study. BMC Med Inform Decis Mak 2001;1:2.

33 Fuller R, Dudley N, Blacktop J. Older people's understanding of cumulative risks when provided with annual stroke risk information. Postgrad Med J 2004;80:677-8.

34 JBS3 Board. Joint British Societies' consensus recommendations for the prevention of cardiovascular disease (JBS3). Heart 2014;100(Suppl 2):ii1-ii67.

35 Freedman B, Potpara TS, Lip GY. Stroke prevention in atrial fibrillation. Lancet 2016;388:806-17.

36 Dufouil C, Beiser A, Chêne G, et al. Are Trends in Dementia Incidence Associated With Compression in Morbidity? Evidence From The Framingham Heart Study. J Gerontol B Psychol Sci Soc Sci 2018;73(Suppl 1):S65-S72.

37 WHO. First WHO Ministerial Conference On: Global Action Against Dementia, 2015.

38 Livingston GSA, Orgeta V. Dementia prevention, intervention, and care. Lancet 2017.

39 National Academies of Sciences E, Medicine, Health. The Health Effects of Cannabis and Cannabinoids: The Current State of Evidence and Recommendations for Research, 2017.

40 Zhang Y, Kivipelto M, Solomon A, et al. Cost-effectiveness of a health intervention program with risk reductions for getting demented: results of a Markov model in a Swedish/Finnish setting. J Alzheimers Dis 2011;26:735-44. 
41 Meschia JF, Bushnell C, Boden-Albala B, et al. Guidelines for the primary prevention of stroke: a statement for healthcare professionals from the American Heart Association/ American Stroke Association. Stroke 2014;45:3754-832.

42 Time RWA, Sex G. History, and Dementia. Alzheimer Dis Assoc Disord 2017;31:76-9.
43 Schrijvers EM, Verhaaren BF, Koudstaal PJ, et al. Is dementia incidence declining?: Trends in dementia incidence since 1990 in the Rotterdam Study. Neurology 2012;78:1456-63.

44 Wu YT, Beiser AS, Breteler MMB, et al. The changing prevalence and incidence of dementia over time - current evidence. Nat Rev Neurol 2017;13:327-39. 